\section{Georg Soldner}

\section{Einleitung}

Akute Atemwegsinfekte (akute Otitis media, Rhinosinusitis, Tonsillopharyngitis, subglottische Laryngitis, Bronchitis; auf die ambulant erworbene Pneumonie kann hier aus Platzgründen nicht eingegangen werden) bilden bei Kindern vor dem Schulalter einen Hauptgrund für die Konsultation eines Kinder- oder Allgemeinarztes in der Praxis und den ärztlichen Notdienst. Das gilt insbesondere, wenn damit Fieber einhergeht, das häufig zum Einsatz von Antiphlogistika führt. Das ist - ausser bei akuten Schmerzen - wenig rational, zumal die meisten Atemwegsinfekte primär viraler Genese sind [1]. Es ist unstrittig, dass die körpereigene Immunabwehr grundsätzlich im Fieber aktiver ist; selbst Antibiotika sind bei Fieber wirksamer als ohne [2]. Fieber stellt einen Zustand gesteigerter Eigenaktivität des Organismus dar. Der Mensch verfügt über die leistungsfähigste Wärmeorganisation aller Lebewesen, und eine rationale Therapie, die am Zuwachs immunologischer Kompetenz des kleinen Kindes interessiert ist, sollte die Fieberreaktion des Organismus eher unterstützen. Diagnostisch ist es sehr schwierig, bei akuten Atemwegsinfekten rasch zu einer verlässlichen Erregerdiagnose zu gelangen. Schwerwiegende Komplikationen wie eine Mastoiditis werden durch eine unkritische Antibiotikaverschreibung jedoch nicht vermindert [3].

Ein zentrales, schwerwiegendes Problem der Medizin ist aktuell die zunehmende Antibiotikaresistenz. Diese stellt wirksame Therapieverfahren, z.B. der Intensivmedizin, Neonatologie oder Onkologie, potenziell zunehmend infrage. Es muss energisch daran

\title{
Akute Atemwegsinfekte im Kindesalter
}

gearbeitet werden, Patienten nicht unnötig Antibiotika und vor allem keine bisher wirksamen Breitbandantibiotika zu verschreiben, die bei stationären, schwer kranken Patienten unverzichtbar sind. Es besteht eine Evidenz dafür, dass es im Rahmen einer integrativen Medizin, die Therapieverfahren der Anthroposophischen Medizin und Homöopathie anwendet, bereits heute gelingt, die Verordnungshäufigkeit von Antibiotika bei akuten Atemwegsinfekten im Kindesalter deutlich unter $10 \% \mathrm{zu}$ senken [4], ebenso den Verbrauch an Antiphlogistika, und gleichzeitig das Outcome gegenüber einer konventionell behandelten Gruppe von Kindern eher zu verbessern (raschere Erholungszeit). Antibiotika haben nachteilige, bei Kleinkindern besonders nachhaltig abträgliche Effekte auf das sich entwickelnde Mikrobiom, was unter anderem eine verzögerte Erholung und ein höheres Allergierisiko so behandelter Kinder erklären kann [5]. Verzichtet der Arzt ausser bei klarem Anhalt für eine schwerwiegende bakterielle Infektion zunächst auf eine antibiotische Therapie («delayed prescription» [3]) und greift in den ersten 3 Tagen zunächst zu bewährten Therapieverfahren, z.B. der Anthroposophischen Medizin und Homöopathie, so kann er damit sowohl dem Kind symptombezogen Hilfe leisten als auch nicht notwendige Antibiotikaverschreibungen auf ein Minimum begrenzen.

\section{Wie wird mein Kind möglichst schnell wieder gesund?}

Wichtig ist es in jedem Fall, zunächst einen guten Kontakt zum Kind und zu seinen Eltern herzustellen. Der Schweregrad einer Infektion wird am ehesten über den Allgemeinzustand des Kindes erfasst. Viele unnötige Verschreibungen erfolgen aus dem Bestreben, vermeintlichen elterlichen Erwartungen gerecht zu werden und die Konsultationsdauer zu minimieren. Wer aber nachhaltig und verantwortungsvoll behandeln will, wird nicht darum herumkommen, den Eltern zunächst die Lage zu schildern (dass «starke Medikamente» meist fehl am Platz sind und das Kind, aber auch die gesamte Medizin schwächen) und ein Therapiekonzept zu erklären, das mit dem sich entwickelnden Immunsystem des Kindes arbeitet. Obwohl beide Seiten heute unter Zeitdruck stehen («Wann kann das Kind wieder in die Krippe?»), ist es nicht so, dass «starke Medikamente» zu weniger Fehlzeiten, weniger Rezidiven und weniger Problemen führen. Im Gegenteil: Würde der Arzt vor jeder Antibiotikaverschreibung die Eltern einfach sachlich über mögliche Nebenwirkungen bis hin zu seltenen schweren Komplikationen aufklären (müssen), würde die Verschreibungsrate mit Sicherheit bereits stark sinken.

\section{Therapiebeginn mit Naturarzneimitteln: Bewährte Vorgehensweisen}

Fussend auf 30-jähriger Erfahrung in Klinik, Klinikambulanz und Praxis [6] sowie auf einem Gemeinschaftsprojekt zur Verschreibung anthroposophischer Arzneimittel mit 210 Kollegen aus 18 Ländern [7] möchte ich im Folgenden knapp Therapiekonzepte zu den häufigsten Atemwegsinfekten (vor allem des Kleinkindalters)

\section{KARGER}

(c) 2017 S. Karger GmbH, Freiburg

Fax +497614520714
Georg Soldner

Facharzt für Kinderheilkunde und Jugendmedizin

Medizinische Sektion am Goetheanum

Postfach, 4143 Dornach, Schweiz

georg.soldner@medsektion-goetheanum.ch 
darstellen. Jede Kollegin und jeder Kollege kann diese in der eigenen Praxis prüfen. Für Eltern haben Jan Vagedes und ich diese Konzepte in leicht verständlicher Form dargestellt [8]. Entsprechend qualifizierte Elternratgeber oder praxiseigene Skripte können eine wertvolle Ergänzung der stets zu kurzen - Konsultationszeit bieten.

\section{Otitis media}

Schon vor dem Arztbesuch kann ein Zwiebelwickel auf das betroffene $\mathrm{Ohr}$ den Schmerz wesentlich erleichtern (Zwiebeln sind potente Schwefelwasserstoff-Spender, was hier zur Wirkung beiträgt). Sollte das nicht der Fall sein, ist dies ebenfalls eine wichtige Information, weil dann mit einer aggressiveren Inflammation zu rechnen ist. Bei starken Schmerzen, blass- bis hochrotem Trommelfell und eventuell Blasen im Sinne einer Grippeotitis habe ich sehr gute Erfahrung mit Apis D4/ Levisticum D3 Globuli WALA gemacht. Anfangs alle 10-15 min 5 Globuli im Mund zergehen lassen, bei Besserung seltener, insgesamt ca. 7 Tage zu geben.

Das Mittel kann ergänzt werden durch Silicea comp. Globuli WALA; täglich anfangs $5 \times$, später $3 \times 5-7$ Globuli.

\section{Rhinosinusitis}

Ein lästiger Säuglingsschnupfen, der das Baby nachts hochfahren lässt, während es kaum Luft bekommt, wird lokal am besten mit Muttermilch und innerlich mit potenziertem Holunder, d.h. Sambucus nigra D4 Globuli, anfangs alle 2 h 3-5 Globuli, dann seltener behandelt.

Virale Infekte der Nase betreffen praktisch immer auch die Nasennebenhöhlen [8]. Hier wie grundsätzlich bei Atemwegsinfekten sind einige allgemeine Regeln wesentlich:

- Wärme: Ist das Kind warm bis in die Peripherie? Im Winter sind Wollbodys hilfreich. Eine Öleinreibung kann wärmend wirken und verbessert bei Frühgeborenen und
Säuglingen das Gedeihen [9]. Bei älteren Kindern (und Erwachsenen) ist ein warmes Fussbad mit Zusatz von 2-3 Handvoll schwarzem Senfmehl oder Ingwerpulver sehr hilfreich bei Rhinosinusitis. Füsse so lange im Wasser lassen, bis die Rötung der Haut erkennbar wird. Nach kurzer Reinigung mit einem neutralen Öl einreiben und ins Bett.

- Ernährung: Bei akuten Infekten keine Süssigkeiten und keinen Zucker geben. Demgegenüber wirken milde Bitterstoffe abwehrsteigernd, während süsse Speisen über den entsprechenden Rezeptor die Aktivierung des Immunsystems schwächen [10]. Lokal kann man klassische Dekongestiva (Xylometazolin usw.) meist vermeiden durch Salzwassernasensprays und eine geeignete Schnupfencreme (z.B. Weleda). Als orale Basismedikation eignen sich wiederum die oben genannten Silicea comp. Globuli (Alternativen sind detailliert in [11] zu finden). Bei aggressiveren, fieberhaften Infekten im Bereich von Nasennebenhöhlen und Mittelohr habe ich gute Erfahrungen gemacht mit Echinacea/Mercurius comp., Suppositorien für Kinder von WALA; $1-3 \times$ täglich 1 Suppositorium.

Dies gilt auch für resistenzgeschwächte Kinder, z.B. mit Morbus Down. Diese Zäpfchen helfen in meiner Erfahrung, Antibiotika einzusparen.

\section{Tonsillopharyngitis}

Der «rote Hals», aber auch die unkomplizierte Tonsillitis stellen - auch bei positivem Nachweis von GABHS (Beta-hämolytische Streptokokken der Gruppe A) - nach aktuellen Leitlinien keine zwingende Antibiotikaindikation dar. Hier kann aus Platzgründen nicht differenziert darauf eingegangen werden. Zurückhaltung bei Antibiotika im Sinne einer «delayed prescription» lohnt sich in den meisten Fällen. Wichtig sind hier Ruhe und gesunde Ernährung; ungünstig ist es, wenn mehrere Familienmitglieder gleichzeitig betroffen sind und auf der
Familie hoher Stress lastet, z.B. durch Umzug oder seelische Belastungen. Im Regelfall kann man die Therapie beginnen mit Apis/Belladonna/Mercurio Globuli WALA, anfangs alle $2 \mathrm{~h}$ 5 (-7) Globuli - bei etwas älteren Kindern ergänzt durch Zinnober D6 Tabletten Weleda, 3-5 $\times$ täglich 0,5 (-1) Tablette im Mund zergehen lassen.

Warme Zitronenhalswickel werden oft als angenehm empfunden.

\section{Subglottische Laryngitis \\ («Pseudo-Krupp»)}

Eine gute Aufklärung und Beruhigung der Eltern ist hier wichtig. Die Schwellung nimmt im Liegen und Schlafen $\mathrm{zu}$; entsprechend tritt die Symptomatik vor allem nachts auf und ist stark angstbesetzt. Ein starkes Angstmittel der Homöopathie in diesen Fällen ist Aconitum D30 Globuli, von dem ich anfangs in vielen Fällen 5 Globuli stündlich gebe. (Todes-)Angst, plötzlicher Beginn abends oder nachts, bellender Husten und eventuell Fieber passen zur Indikation dieses Mittels.

Ein sehr sicher wirkendes Mittel bei Pseudokrupp ist Bryonia/Spongia comp., Weleda. Man verabreicht anfangs alle 10-15 min eine Gabe, und Beruhigung tritt in der Regel innerhalb von 30-45 min ein. Kinder unter 3 Jahren erhalten 15-25 Tropfen/Tag in einem halben Glas Wasser, davon pro Gabe ein kleiner Schluck; über 3 Jahre alte Kinder nehmen 5 Tropfen pro Dosis ein.

\section{Bronchitis}

Die obstruktive Bronchitis und Bronchiolitis (wie die Pneumonie) sprengen den hier gesetzten Rahmen. Die einfache Tracheobronchitis mit Husten und Fieber kann gut mit äusseren Anwendungen behandelt werden. Hilfreich sind warme Ölwickel mit 10\% Lavendelöl bei trockenem Husten und gestörtem Schlaf. Quarkwickel (Magerquark, $3 \mathrm{~min}$ bei vorgeheiztem Backrohr $\left(100^{\circ} \mathrm{C}\right)$ schonend erwärmt auf dem Wickeltuch, nach Temperaturkontrolle um die Brust an- 
gelegt mit zwei saugfähigen, breiteren äusseren Wickeltüchern) lassen die Kinder gut schlafen und sind bei feuchter und auch feucht-obstruktiver Bronchitis im Kleinkindalter wirksam. Handelt es sich um ein Kind, das fiebert, zur Auskühlung neigt und vielleicht schon einmal eine Pneumonie durchgemacht hat, empfehle ich einen fachgerecht durchgeführten Ingwerbrustwickel [7, Kapitel 5.1].

\section{Literatur}

1 Dippold N: Leitliniengerechte Therapie von Schnupfen und Sinusitis. MMW Fortschritte der Medizin 2017;159:37-40.

2 Martin D: Fever: views in anthroposophic medicine and their scientific validity. Evid Based Complement Alternat Med 2016;2016:3642659.

3 Simon A: Antibiotic Stewardship in der kinderärztlichen Praxis. Kinder- und Jugendarzt 2016;47:51-57.

4 Jeschke E, Lüke C, Ostermann T, Tabali M, Hübner J, Matthes H: Verordnungsverhalten anthroposophisch orientierter Ärzte bei akuten Infektionen der oberen Atemwege. Forsch Komplementärmed 2007;17:207-215.
Kräutertee (Spitzwegerich, Malvenblüten, Eibischwurzel und andere), dem trinkwarm etwas Honig zugesetzt wird, ersetzt viele Hustensäfte.

Für die innere Anwendung existiert eine breite Vielfalt von Hustenmitteln. Hervorheben möchte ich bei dieser Indikation in der kalten Jahreszeit, besonders bei «anfälligen», in ihrer Wärme labilen Kindern: Infludoron Globuli Weleda; anfangs alle 2 h 5-10
Globuli, dann seltener und nicht länger als 3-5 Tage.

Bei jeder Bronchitis, die zur Obstruktion neigt, eignen sich Petasites comp. Glob. WALA, 15-40 Globuli/ Tag in Wasser gelöst schluckweise über den Tag verteilt.

Eine detaillierte Darstellung zu Krankheitsverständnis und Therapie findet der Leser in dem Buch «Individuelle Pädiatrie» [7].
5 Marra F, Lynd L, Coombes M, Richardson K, Legal M, Fitzgerald JM, Marra CA: Does antibiotic exposure during infancy lead to development of asthma? Chest 2006;129:610-618.

6 Soldner G, Stellmann HM: Individuelle Pädiatrie, ed 4. Stuttgart, WVG, 2011.

7 Gesellschaft Anthroposophischer Ärzte in Deutschland: Vademecum Anthroposophische Arzneimittel, ed 3. Berlin, Der Merkurstab, 2013.

8 Vagedes J, Soldner G: Das Kindergesundheitsbuch, ed 6. München, Gräfe und Unzer, 2016.
9 Salam RA, Das JK, Darmstadt GL, Bhutta ZA: Emollient therapy for preterm newborn infants - evidence from the developing world. Public Health 2013;13(suppl 3):S31.

10 Cleminson J, McGuire W: Topical emollient for preventing infection in preterm infants. Cochrane Database Syst Rev 2016;(1):CD001150.

11 Lee R, Kofonow JM, Rosen PL, et al: Bitter and sweet taste receptors regulate human upper respiratory innate immunity. J Clin Invest 2014; 124:1393-1405. 